\title{
Cooperation in the snowdrift game on directed small-world networks under self-questioning and noisy conditions
}

\author{
Tian Qiu ${ }^{\mathrm{a}, *}$, Tarik Hadzibeganovic ${ }^{\mathrm{b}}$, Guang Chen ${ }^{\mathrm{a}}$, Li-Xin Zhong ${ }^{\mathrm{c}}$, Xiao-Run $\mathrm{Wu}^{\mathrm{a}}$ \\ ${ }^{a}$ School of Information Engineering, Nanchang Hangkong University, Nanchang, 330063, China \\ ${ }^{b}$ Cognitive Science Section, Department of Psychology, University of Graz, 8010 Graz, Austria \\ ${ }^{c}$ School of Journalism, Hangzhou Dianzi University, Hangzhou, 310018, China
}

\begin{abstract}
Cooperation in the evolutionary snowdrift game with a self-questioning updating mechanism is studied on annealed and quenched small-world networks with directed couplings. Around the payoff parameter value $r=0.5$, we find a size-invariant symmetrical cooperation effect. While generally suppressing cooperation for $r>0.5$ payoffs, rewired networks facilitated cooperative behavior for $r<0.5$. Fair amounts of noise were found to break the observed symmetry and further weaken cooperation at relatively large values of $r$. However, in the absence of noise, the self-questioning mechanism recovers symmetrical behavior and elevates altruism even under large-reward conditions. Our results suggest that an updating mechanism of this type is necessary to stabilize cooperation in a spatially structured environment which is otherwise detrimental to cooperative behavior, especially at high costto-benefit ratios. Additionally, we employ component and local stability analyses to better understand the nature of the manifested dynamics.
\end{abstract}

Keywords: Evolution of cooperation, snowdrift game, networks, small-world, Monte-Carlo simulation, agent-based model.

\section{Introduction}

Understanding the ubiquity of cooperative behavior in complex biological, technological, social, and economic networks has become one of the central research topics in the past years in a variety of disciplines, including physics [1, 2, 3, 4]. In the course of this challenging process of scientific enquiry, the evolutionary game theory emerged as a common mathematical framework for investigating how and why individuals happen to overcome selfish behavior in order to help others and to contribute to the common good [5, 6, 7, 8, 9, 10, 11, 12, 13].

Within this framework, two widely discussed games are the Prisoner's Dilemma (PD) and the Snowdrift (SD) Game, where in both cases, the bilateral cooperation yields the highest collective payoff, equally distributed between the interacting play'ers [6]. However, providing help to a neighboring individual is challenged by the presence of an alternative defecting strategy, that warrants the defector a higher benefit at the expense of a cooperating agent.

The major difference between the PD and SD games is manifested in the actual ranking of the payoff values, i.e. in the manner by which defecting agents are punished when facing one another. In the PD game, a defecting agent facing another defector earns more than a cooperator, while in the SD game, which can be seen as a modified PD game, mutual defection gives the lowest payoff. We therefore have the reversed situa-

${ }^{*}$ School of Information Engineering, Nanchang Hangkong University, Nanchang, 330063, China

Email address: tianqiu.edu@gmail.com (Tian Qiu ) tion where a cooperator obtains a higher payoff than a defector when the latter interacts with another defecting agent.

Thus, while in the PD game we have $T>R>P>S$ ranking of the payoffs, for the SD game this ranking is given by $T>$ $R>S>P$. Here, the payoff for bilateral cooperation is denoted with $R$, whereas $P$ represents the payoff for mutual defection. $T$ is the temptation to defect by one of the two interacting sides, resulting in the payoff $S$ (the "sucker's payoff") for the other, cooperating player.

At first, this apparently minor difference between the two payoff rankings might seem as rather unimportant, however, it has been demonstrated [7, 8] that it can have a fundamental impact on the final evolutionary outcome of a studied system in the sense of altering the actual level of cooperative behavior whenever interacting individuals are facing a social dilemma.

Whereas investigations on simple regular lattices yielded far more converging results, studies of PD and SD games on complex networks are still rather inconclusive about the overall structural effects on cooperation [1, 10]. Earlier reports suggested that spatial structure generally tends to facilitate cooperative behavior [8, 14, 15]. Indeed, the scale-free topology [16] was found to be consistently beneficial for overcoming selfish behavior in both PD and SD games [17]. However, there have been many opposite findings in recent years in other than scalefree topologies [7, 18, 19, 20, 21]. In addition, a number of special conditions for the elevation and inhibition of cooperation in both game types have been identified [22, 23].

Prompted by the observation that structured SD and PD games often result in different densities of cooperative agents under different connection topologies [7, 8, 14, 15, 24], an ever 
increasing effort has been dedicated to a better understanding of the mechanisms involved in this 'spatial selection' process $[10,41,17,25,26,27,28,[29,30,31,32,33]$. Representative examples are the pertaining studies of the emergence and survival of cooperation on directed networks [17, 34, 35, 36]. Directed interaction [37, 38, 39, 40], found in many social and economic systems, implies here that an agent influencing its nearest-neighbor may not be influenced by this neighbor in return. Thus, the influence spreads in one direction only.

Besides the effects of topology, it has been demonstrated earlier that the peculiarities of strategy update rules in theoretical social dilemma models can play a decisive role in shaping the evolutionary dynamics of cooperation [41, 42, 43]. Different strategy updating rules have been studied so far, for example, the widely applied deterministic rule proposed by Nowak and May [8, 24], and the stochastic evolutionary rule by Szabó and Töke [44].

Motivated by the yet understudied effects of directed network topologies and alternative updating rules, the scope of the present paper is to identify the pertinent mechanisms leading to cooperative behavior in SD-type of interactions on annealed and quenched small-world networks [45, 46] with directed links. A special attention is given to an alternative strategy updating rule, the so-called self-questioning mechanism [32, 47], and its influence on cooperative behavior under noisy conditions.

In the course of self-questioning, players typically compare their current and the opposite strategies, and then eventually adopt a more advantageous strategy with a certain probability. The self-questioning mechanism can therefore be seen as a kind of a self-evaluation procedure that helps in maximizing the benefits of interacting agents.

To prevent deterministic behavior of the model and allow for irrational decision making, we study the effects of noise, implemented here as a low probability with which an agent shifts from its own strategy to the strategy of a randomly chosen neighbor. Considering such noise effects in models of social dynamics [48, 49, 50, 51] might be useful for a better understanding of the influence of bounded rationality in evolutionary snowdrift and other games [52]; see also Ref. [53] for cooperation under noisy conditions in the PD game and Ref. [54] for noise effects in both PD and SD games. Finally, we employ an extensive local stability analysis inspired by the Ref. [32], and also implement a component analysis, in order to better understand the nature of the observed cooperative behavior.

The remainder of this paper is organized as follows. In the next section, we detail the model structure and the associated game dynamics. In Section 3, we outline the simulation results and investigate the possible origin of the manifested cooperative behavior. In Section 4, we analyze the influence of the introduced strategy updating rule on cooperation under different reward and noisy conditions. Section 5 describes analytic methods useful for a further comprehension of the obtained results. Finally, we conclude and suggest further research directions in Section 6.

\section{Model}

In the present paper, all simulations are carried out for the snowdrift (SD) game which we first outline below and then describe the simulation setup.

In the SD game [55], players obtain a payoff $R=b-c / 2$ when they both cooperate, and a payoff $P=0$ when they both defect. When one player cooperates but the other one defects, the defector gets the payoff $T=b$, and the cooperator receives the payoff $S=b-c$. The SD game refers to the case $b>$ $c>0$, leading to the payoff ranking $T>R>S>P$. For simplicity, we assign $R=1$ to characterize all payoffs by a single parameter $r=c / 2=c /(2 b-c)$, defined as the cost-tobenefit ratio. The payoffs are then rewritten as $T=1+r, R=1$, $S=1-r$, and $P=0$, or, when shown in a rescaled payoff matrix

$$
\begin{gathered}
C \\
D
\end{gathered}\left(\begin{array}{cc}
1 & D \\
1+r & 0
\end{array}\right)
$$

where $0<r<1$. Each matrix element represents here the payoff of a player selecting a strategy from the left hand column when the opponent selects a strategy listed in the top row.

In the initial configuration of our model, all players occupying the network sites are assigned a strategy, either as a cooperator (C) or as a defector (D), with equal probability. Thus, we assume that each vertex of a network, ranging from a regular $L \times L$ square lattice to a random graph by modifying $p$, is populated by a player choosing just cooperation or defection.

Following the initial distribution of strategies, player interactions and strategy updates are repeated in an iterative fashion in accordance with the Monte Carlo (MC) simulation method. Size-wise, simulations are conducted on networks with $L=$ $100, L=300$, and $L=500$. Furthermore, we separately investigate the model behavior evolving on quenched and annealed small-world networks. More specifically, the underlying evolutionary dynamics are generated by the following elementary events:

(1) We first construct a small-world network with directed couplings [56]. Starting with an $L \times L$ regular lattice, where each node is bidirectionally connected with its four neighbors, we rewire with probability $p$ each of the outward links to another randomly chosen node.

(2) At each time step, players occupying the nodes of a directed small-world network of a size $L^{2}$ obtain payoffs on the basis of the above described payoff matrix by interacting with all outward-connected neighbors in accordance with the SD game rules. A player $i$ obtains a payoff $\overline{V_{i}}=V_{i} / 4$ by interacting with its four outward-linked neighbours, where $V_{i}$ is obtained by summing up the payoffs of all outward-linked neighbors. Subsequently, by means of self-questioning in a virtual game [32, 47], each player adopts its anti-strategy and estimates a virtual payoff $\overline{V_{v}}=V_{v} / 4$, where $V_{v}$ is obtained by summing up the virtual payoffs of all the outward-linked neighbors. By comparing the virtual payoff with the real payoff, players can estimate their optimal strategy corresponding to the highest payoff and realize whether their current strategy is of any advantage [47]. 
Specifically, player $i$ will shift from its current strategy to its anti-strategy with the strategy transition probability $w$, defined as the normalized difference between the estimated virtual payoff $\overline{V_{v}}$ and the actual real payoff $\overline{V_{i}}$ :

$$
w=\frac{\overline{V_{v}}-\overline{V_{i}}}{1+r}
$$

where $1+r$ in the denominator is only a normalization factor [31], and as such, does not affect the results reported in the subsequent sections. If $w>0$, then the current strategy of player $i$ is updated with probability $w$, i.e. replaced by the more advantageous anti-strategy; otherwise, it remains unchanged.

(3) Network nodes are randomly scanned until each one is selected once and then the rewiring process of step (1) and the strategy evolution of step (2) are repeated.

Given these 3 outlined steps, we stress here that we investigate a total of two different cases, i.e., 1) simulations on quenched and 2) annealed small-world networks.

In the first case, we assume that after a directed small-world network is once established, the system evolves based on iterating the step (2). Here the SD game dynamically evolves on the static topology of the network with a fixed link directedness, which we here call the quenched network [45, 57].

In the second case, we consider the system evolution based on repeating all outlined steps, i.e. the steps (1), (2) and (3). Thus, after initially constructing a directed small-world network and then following the update of strategies in accordance with step (2), all nodes of the network are subsequently scanned and the rewiring process of step (1) is re-initiated, leading thereby to a different realization of rewired links. The process is then further cycled with steps (2) and (3) following the step (1). In this particular case, the SD game is said to evolve on an annealed network [37, 45, 46].

Finally, we note here again that in the SD game, the best action for a player is to defect if the opponent cooperates, but otherwise it is better to opt for cooperation. However, larger values of the payoff parameter $r$ in the SD game generally tend to encourage defection [7, 32]. We therefore investigate what is the critical value of $r$ for and under which cooperation is still promoted given the specified structural and strategy update characteristics of our model. Thus, it is important to investigate how the frequency of cooperators $f_{c}$ dynamically relies on the payoff parameter $r$ and on the spatial structure, i.e. the rewiring probability $p$ of the network (where $f_{c}$ is the ratio of the number of cooperators and the total number of individuals in a population).

\section{Evolution of cooperation as a function of the spatial structure $\left(f_{c}\right.$ vs. $\left.p\right)$}

\section{1. $f_{c}$ vs. $p$ on quenched and annealed networks}

In Fig. 1(a) and (b), we show $f_{c}$ as a function of $p$ for quenched and annealed networks with directed couplings. We observe a symmetrical cooperation behavior around the payoff parameter value $r=0.5$ for both networks. Cooperation dominates for $r<0.5$, but is significantly suppressed for $r>0.5$ as $p$ increases. For $r=0.5, f_{c}$ remains stable for a wide range of values of $p$. The displayed results in Fig. 1 are the outcomes of simulations conducted on networks with $100 \times 100$ nodes. Simulations on larger networks $(300 \times 300$ and $500 \times 500)$ did not yield significantly different results (not shown).
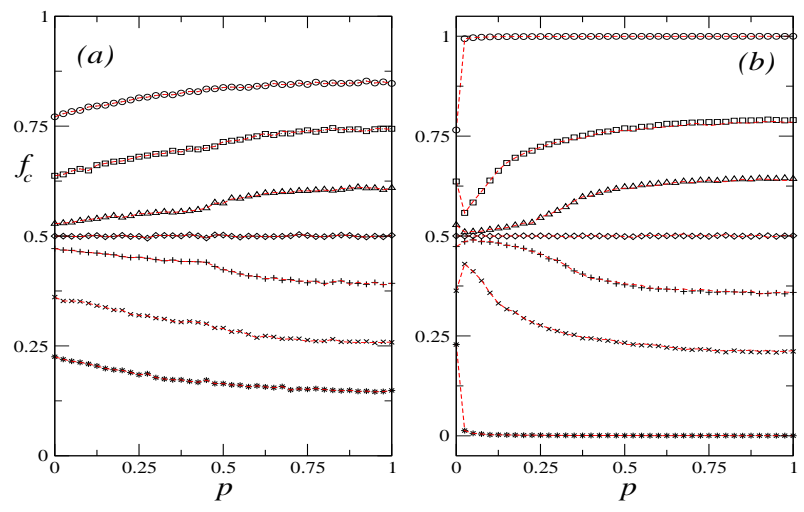

Figure 1: The frequency of cooperators $f_{c}$ as a function of the rewiring probability $p$ is displayed in (a) and (b), with circles, squares, triangles, diamonds, pluses, crosses and stars standing for (from top to bottom) $r=0.0,0.1,0.3,0.5$, 0.7, 0.9 and 1.0. (a) shows the quenched network and (b) the annealed network. The red dashed curves are the theoretical fits.

For an intuitive understanding of the evolutionary behavior of the system, we investigated the spatial patterns associated with different values of $p$ and $r$. In Fig. 2, we illustrate the typical patterns emerging on a quenched small-world network with directed links. The annealed network shows similar behavior (here not shown). The displayed patterns are independent of the initial configuration and statistically static after the system reaches the steady state. In Fig. 2(a), cooperating and defecting agents are scattered over the lattice in a chessboard-like fashion for the parameter values $p=0.2$ and $r=0.2$, i.e. sites with one strategy are roughly regularly surrounded by the sites with the opposite strategy.

We then increase the rewiring probability up to $p=0.8$ while keeping the payoff value $r=0.2$ unchanged (Fig. 2(b)). It can be observed here that at fixed and low $r$, cooperators start to form clusters as the rewiring probability increases. In contrast, when increasing and fixing the payoff parameter value at $r=0.8$, and again modifying $p$ from $p=0.2$ to $p=0.8$, the observable strategy pattern transforms from a state of scattered cooperators and defectors into the state of clustered defectors (Fig. 2(c) and (d)). This is consistent with previous findings showing that low $r$ stimulates cooperative behavior, whereas elevated $r$ values tend to hinder the evolution of cooperation [7, 32].

\subsection{The effects of inward-link heterogeneity and long-range in- teraction}

To clarify whether the inward-link heterogeneity influences cooperation, one can remove it by artificially fixing the number of the inward links $k_{i n}$. If cooperative behavior remains qualitatively unchanged after such a modification, one can then 


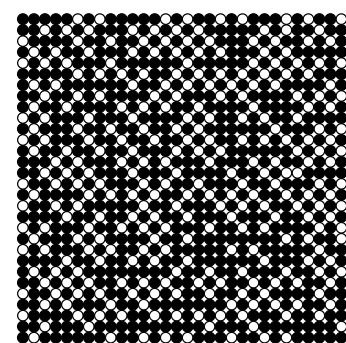

(a) $p=0.2, r=0.2$

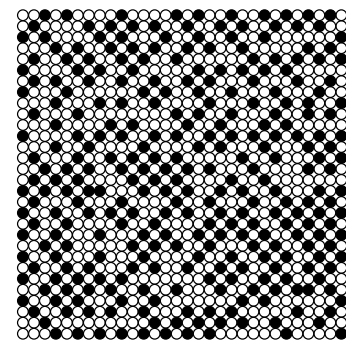

(c) $p=0.2, r=0.8$

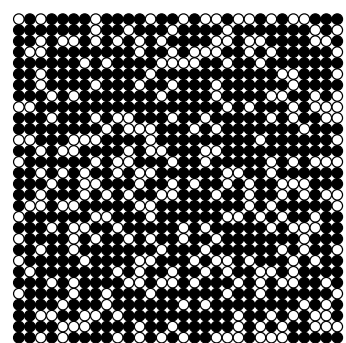

(b) $p=0.8, r=0.2$

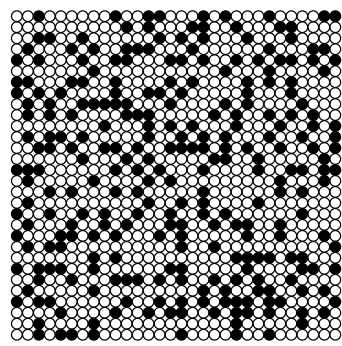

(d) $p=0.8, r=0.8$
Figure 2: Representative simulation snapshots for the spatial snowdrift game after a sufficiently long transient time. The results are shown for a $30 \times 30$ portion of the full $100 \times 100$ lattice with typical spatial patterns emerging via agent interactions on the quenched network, with $\mathrm{C}(\mathrm{D})$ nodes displayed as solid(open) circles. (a) is for $p=0.2$ and $r=0.2$, (b) for $p=0.8$ and $r=0.2$, (c) for $p=0.2$ and $r=0.8$, and (d) for $p=0.8$ and $r=0.8$.

eliminate the possible influence of inward-links. Taking again the quenched network as an example, in Fig. 3(a) we show $f_{c}$ as a function of $p$ with fixed inward-links as $k_{i n}=4$, which corresponds to a regular lattice. We see that $f_{c}$ again shows a familiar symmetrical behavior, as without this link fixation. Moreover, we find that $f_{c}$ remains nearly steady for many different values of $k_{i n}$, as shown in Fig. 3(b). Additionally, we found that also the annealed network simulations with fixed inward-links resulted in a qualitatively similar behavior.
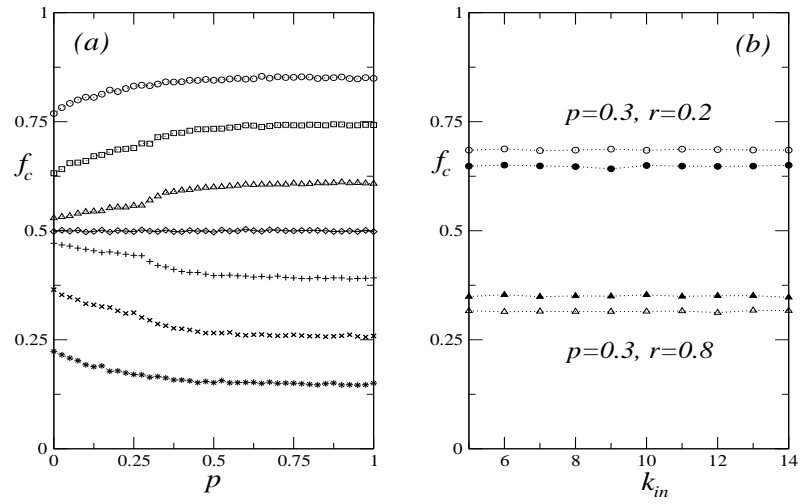

Figure 3: (a) The frequency of cooperators $f_{c}$ as a function of the rewiring probability $p$ is displayed for the quenched network with a fixed number of inward-links $k_{i n}=4$. Circles, squares, triangles, diamonds, pluses, crosses and stars correspond to the payoff parameter values (from top to bottom) $r=0.0,0.1$, $0.3,0.5,0.7,0.9$ and 1.0. (b) $f_{c}$ as a function of $k_{\text {in }}$ for a rewired probability $p=$ 0.3 , and with open (solid) circles standing for $r=0.2$ and open (solid) triangles for $r=0.8$, both simulated on quenched (annealed) networks, respectively.

To further understand the origin of the symmetrical cooperation observed in Fig.1, we study the possible effect of long-range interactions by analyzing the model evolution on quenched and annealed small-world networks with undirected links. As shown in Fig. 4(a), the rewiring process has little effect on the cooperation level for the undirected quenched network. However, the previously discovered symmetrical behavior remains robust in this undirected couplings version, under both quenched and annealed network regimes.

Having eliminated the long-range interaction and the inwardlink heterogeneity effects on the symmetrical behavior, we ask whether it is the nature of the implemented strategy updating rule (generalized self-questioning mechanism) that is actually causing the observed cooperation symmetry. We address this issue in a more detail in the subsequent section.
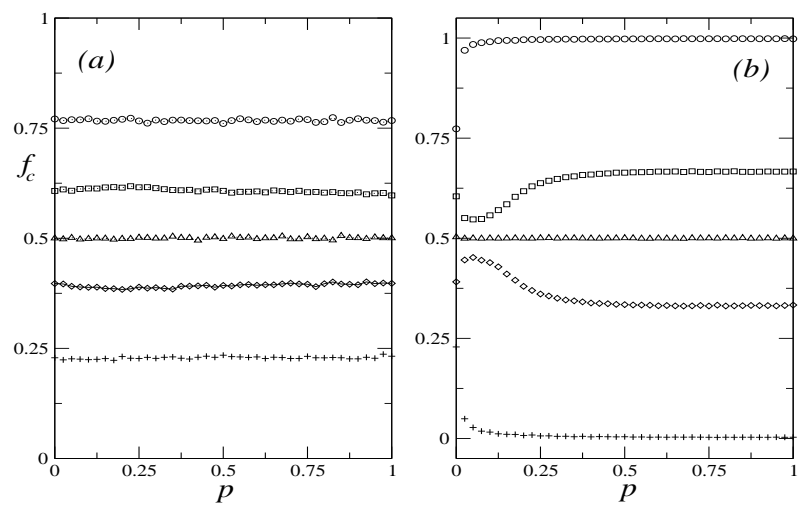

Figure 4: The frequency of cooperators $f_{c}$ as a function of the rewiring probability $p$ in (a) quenched and (b) annealed undirected small-world networks. Circles, squares, triangles, diamonds and pluses correspond to (from top to bottom) $r=0.0,0.25,0.50,0.75$, and 1.00 .

\subsection{The effect of noise}

To prevent the purely deterministic behavior and to enable irrational choices, we introduced noise $w_{\text {noise }}$ in the model while keeping the model topology unchanged, i.e., the noise effects were studied on quenched and annealed small-world networks with directed links. More specifically, randomness was implemented as a low probability with which a player shifts from its own strategy to the strategy of a randomly chosen neighbor. Surprisingly, we find that only after this direct modification of the previously implemented strategy update rule the results started to change (see Fig. 5). In particular, the observed cooperation symmetry is broken after directly influencing the strategy updating mechanism via noise. These changes are visible already after adding a rather small amount of noise $w_{\text {noise }}=0.05$, but a clearly more pronounced effect is observed for $w_{\text {noise }} \geq 0.1$ (Fig. 5).

Taken together, these results suggest that the symmetrical behavior of $f_{c}$ arises due to the characteristics of the implemented game rule, i.e., due to the so-called self-questioning mechanism [32, 47] which drives the process of strategy updating in our model. In contrast, enabling irrational choice behavior via noise breaks the cooperation symmetry, however, it is unclear yet whether the self-questioning mechanism can support cooperative clusters against the destructive effect of 

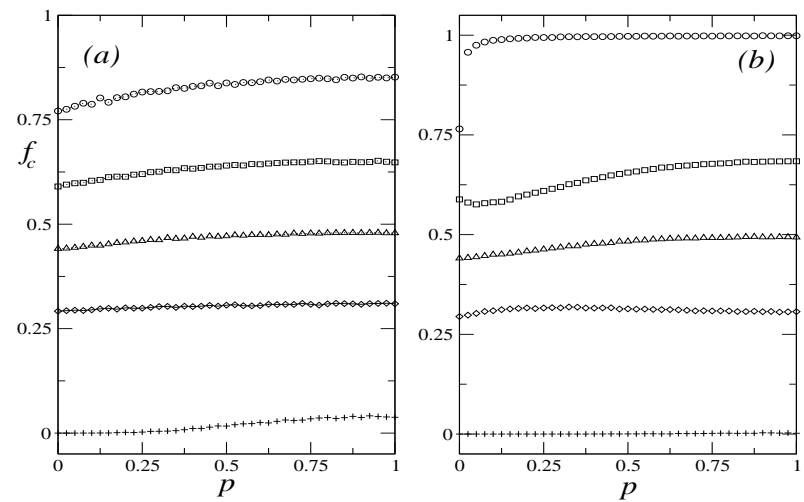

Figure 5: The effect of noise $w_{\text {noise }}=0.1$ on the behavior of (a) quenched and (b) annealed small-world networks with directed couplings. Circles, squares, triangles, diamonds and pluses correspond to (from top to bottom) $r=0.0$, $0.25,0.50,0.75$, and 1.00 .
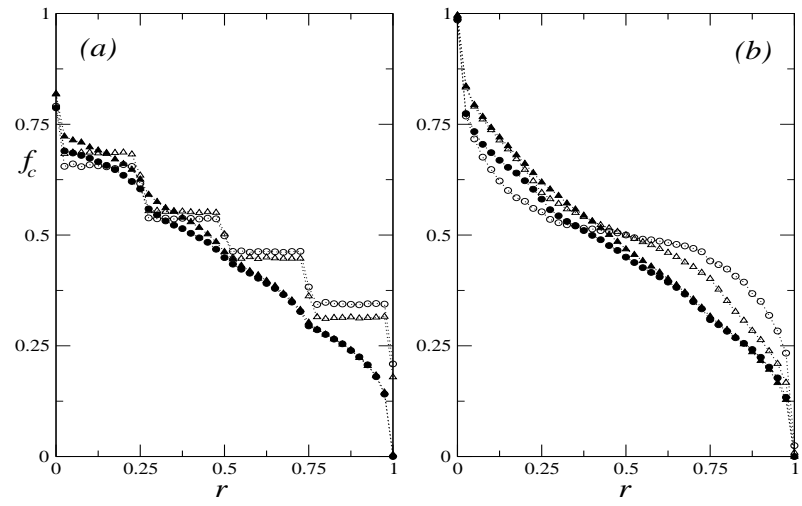

Figure 6: The fraction of cooperators $f_{c}$ as a function of $r$ is displayed. (a) shows the results for the quenched and (b) for the annealed network. The open circles and the triangles correspond to simulation results with the selfquestioning mechanism without noise, and with the rewiring probabilities $p=0.1$ and $p=0.3$, respectively. The solid circles and triangles correspond to simulation outcomes obtained under noisy conditions, with the noise parameter value set at $w_{\text {noise }}=0.1$ and the rewiring probabilities $p=0.1$ and $p=0.3$, respectively.

noise also under large-reward conditions, i.e. at high cost-tobenefit ratios. This is investigated in detail in the following section.

\section{Dependence of $f_{c}$ on $r$}

\subsection{The self-questioning mechanism without noise}

We now investigate the fraction of cooperators $f_{c}$ as a function of the payoff parameter $r$ in the SD-game on quenched and annealed small-world networks with an implemented generalization of the self-questioning mechanism described in Section 2. Here we care more about the evolution of cooperation at a relatively small value of the rewiring probability $p$, because larger $p$ typically results in too many random realizations of long-range interactions among interacting players.

As shown in Fig. 6 (open circles and triangles), $f_{c}$ is observed to decay with $r$ both in quenched and annealed networks with directed couplings. More interestingly, step-wise decay of cooperation not seen in the annealed network and sharp transitions at the critical points $r_{c}=0.25,0.50$ and 0.75 are observed for the quenched small-world network.

The decay dependence of $f_{c}$ on $r$ can be explained analytically from the payoff matrix shown in Table 1, where the total transition probabilities $W_{C \rightarrow D}$ from $\mathrm{C}$ to $\mathrm{D}$ and $W_{D \rightarrow C}$ from $\mathrm{D}$ to $\mathrm{C}$ can be calculated as $4-\frac{11}{2(1+r)}$ and $-4+\frac{13}{2(1+r)}$; they are monotonously increasing and decaying with $r$, respectively.

To understand the critical transition points $r_{c}$, we apply an extensive local stability analysis, following the method detailed in Ref. [32]. At each critical point $r_{c}$, the payoff of a cooperating player should equal that of a defector. The local stability equation is then written as

$$
m+\left(k_{\text {out }}-m\right)\left(1-r_{c}\right)=\left(1+r_{c}\right) m
$$

where $k_{\text {out }}$ is the number of the out-going links, with $k_{\text {out }}=4$ in our model, and $m$ is the number of $C$ neighbours. One can get $r_{c}=\left(k_{\text {out }}-m\right) / k_{\text {out }}$, and therefore obtain $r_{c}=0.25,0.50$ and 0.75 , which is in excellent agreement with the obtained simulation results.

\subsection{Self-questioning under noisy conditions}

To further understand the nature of the observed cooperation in the SD-game on complex networks with the self-questioning mechanism, we investigate the evolution of $f_{c}$ as a function of $r$ when the strategy updating is disturbed by noise, i.e. by the introduction of random choices. As can be seen in Fig. 6 (solid circles and triangles), the level of cooperation in the total population of agents decreases as a function of $r$, also in the presence of noise. Slight concavities of the solid circles and triangles are visible as remnants of the previously observed critical points $r_{c}$ in the quenched network, however, the previous step-wise decay is now almost totally smoothed out by noise.

Furthermore, we see that a fair amount of noise generally hinders the evolution of cooperation for a wide range of values of $r$ (especially for larger $r$ ), and this holds true for both quenched and annealed networks. However, in the absence of noise, the process of repeated self-questioning can re-establish symmetrical cooperation behavior and elevate altruism among interacting agents even under large values of $r$ (see the curves with open circles and triangles in Fig. 6). These results suggest that self-questioning can serve as a highly useful cooperation supporting mechanism in noise-free environments, but is not robust enough against the destructive effects of noise-driven random choices.

\section{Component Analysis}

To further understand the evolution of cooperation in our model, we apply a theoretical analysis, which we here call the component analysis. We first specify that each player occupying a network vertex can have a total of five possible neighborhood configurations. The payoff matrix can then be analytically obtained from the strategy update rules, as shown in Table1. $E_{C}$ and $E_{D}$ are the payoffs of the cooperating (C) and defecting (D) players, $w_{C \rightarrow D}$ is the transition probability with which a player 
Table 1: The payoff matrix in the SD game with a generalized self-questioning mechanism. From left to right, the columns correspond to the neighborhood configuration, the payoff of the $\mathrm{C}$ player $E_{C}$, the payoff of the D player $E_{D}$, the probability of transition from $\mathrm{C}$ to $\mathrm{D} w_{C \rightarrow D}$, and the probability of transition from $\mathrm{D}$ to $\mathrm{C} w_{D \rightarrow C}$, respectively.

\begin{tabular}{|c|c|c|c|c|}
\hline & $E_{C}$ & $E_{D}$ & $w_{C \rightarrow D}$ & $w_{D \rightarrow C}$ \\
\hline C C C C & 1 & $1+r$ & $1-\frac{1}{1+r}$ & 0 \\
C C C D & $1-\frac{r}{4}$ & $\frac{3}{4}+\frac{3 r}{4}$ & $1-\frac{5}{4(1+r)}$ & $-1+\frac{5}{4(1+r)}$ \\
C C D D & $1-\frac{r}{2}$ & $\frac{1}{2}+\frac{r}{2}$ & $1-\frac{3}{2(1+r)}$ & $-1+\frac{3}{2(1+r)}$ \\
C D D D & $1-\frac{3 r}{4}$ & $\frac{1}{4}+\frac{r}{4}$ & $1-\frac{7}{4(1+r)}$ & $-1+\frac{7}{4(1+r)}$ \\
D D D D & $1-r$ & 0 & 0 & $-1+\frac{2}{1+r}$ \\
\hline
\end{tabular}

shifts from $\mathrm{C}$ to $\mathrm{D}$, and $w_{D \rightarrow C}$ is the the transition probability for shifting from $\mathrm{D}$ to $\mathrm{C}$. The frequency of cooperators $f_{c}$ is then written as

$$
f_{c}=\sum_{i=1}^{5} g_{C}^{i} w_{C \rightarrow C}^{i}+g_{D}^{i} w_{D \rightarrow C}^{i}
$$

where $i=1, \ldots, 5$ labels the corresponding neighbour configurations shown in the leftmost column of the Table $1 . g_{C}^{i}$ and $g_{D}^{i}$ are the proportions of the $\mathrm{C}$ and D players in the system, with $\sum_{i=1}^{5}\left(g_{C}^{i}+g_{D}^{i}\right)=1$. $w_{C \rightarrow C}^{i}$ is the probability that $C$ players do not change their strategy, with $w_{C \rightarrow C}^{i}=1-w_{C \rightarrow D}^{i}$. Due to the difficulty of obtaining the $g_{C}^{i}$ and $g_{D}^{i}$ analytically, we compute these proportions from numerical simulations. As shown in Fig. 1(a) and (b) with the red dashed curves, the theoretical fits of $f_{c}$ vs. $p$ are in a very good agreement with those obtained from numerical simulations.

\section{Conclusions}

In the present paper, we have studied the evolutionary SDgame with a generalized self-questioning updating mechanism. The model was investigated on both annealed and quenched small-world networks with directed couplings. Agent-based computer simulations and semi-analytic results have been presented.

We found that the observed symmetrical cooperation effect around the payoff parameter value $r=0.5$ was size-invariant and independent from both the inward-link heterogeneity and long-range interactions in the studied small-world network. Moreover, our results suggest that the self-questioning updating mechanism might be necessary to stabilize cooperation in a spatially structured environment which is otherwise not beneficial or even detrimental to cooperative behavior. Our results thus support the previous findings showing that spatial structure should not necessarily be seen as a general promoter of cooperation [7], and that one should rather avoid the conceptual flaws of many recent studies according to which an implemented topology automatically enhances cooperative behavior by default.

Compared with the previous work in this field [32, 47], our version of the self-questioning mechanism showed clear advan- tages in (re)establishing and sustaining symmetrical cooperative behavior. However, we found that the cooperation promoting effects of the implemented updating rule were not robust enough under noisy conditions. On the other hand, in the absence of noise, the self-questioning mechanism was able to recover elevated altruism even at large cost-to-benefit ratios. We therefore argue that our updating mechanism can function as an efficient cooperation elevator in complex environments where the impact of noise is insignificant or totally absent.

In the presented model, the rewiring of the network was independent from the actual cooperation status of the individual network nodes. It would therefore be much more interesting in future studies to let links between two rarely cooperating nodes vanish with higher probability than links between frequently cooperating nodes [58]. In future investigations one should also employ the simulated annealing method [59], in order to better avoid the local extrema trap, and to enable the system to get into the global extremum.

As suggested in Ref. [60], future computer simulations should also explore networks with weighted links, since in the present paper all network connections were equally strong. However, in real social networks, people typically have many initial connections, but after a sufficient time, only a few of them survive as strong and important [10,61].

Another line of future generalizations that could potentially make our model more realistic would include simulations with reproducing agents [36], different migratory behaviors of interacting individuals [50, 53], bottleneck and ageing effects on cooperation [62], or the influence of the approaching extinction of a studied population [63].

In sum, presented results demonstrate that under noise-free conditions, the self-questioning updating mechanism can become advantageous for cooperation in the evolutionary snowdrift game on both annealed and quenched small-world networks with directed links. It remains therefore a challenge for future studies to search for advanced modifications of the introduced updating rule which could then fully resist the destructive effects of noise.

\section{Acknowledgments:}

We are grateful to Prof. Dayin Hua for fruitful discussions on a variety of topics related to the present study. We are especially thankful to Prof. Dietrich Stauffer for many useful suggestions and comments on a previous version of the present paper. This work was partly supported by the National Natural Science Foundation of China (Grants No. 10805025, 10774080), Jiangxi Provincial Educational Foundation of China under Grant No. GJJ08231, and Zhejiang Social Sciences Association under Grant 08N51.

\section{References}

[1] M. A. Nowak, C. E. Tarnita, T. Antal, Phil. Trans. R. Soc. B 365 (2010) 19.

[2] F. C. Santos, J. F. Rodrigues, J. M. Pacheco, Proc. R. Soc. B 273 (2006) 51.

[3] C. Castellano, S. Fortunato, V. Loreto, Rev. Modern Phys. 81 (2009) 591. 
[4] F. Schweitzer, G. Fagiolo, D. Sornette, F. Vega-Redondo, A. Vespignani, D. R. White, Science 325 (2009) 422.

[5] R. Axelrod, W. D. Hamilton, Science 211 (1981) 1390.

[6] R. Axelrod, The Evolution of Cooperation, Basic Books, New York, 1984.

[7] C. Hauert, M. Doebeli, Nature 428 (2004) 643.

[8] M. A. Nowak, R. M. May, Nature 359 (1992) 826.

[9] E. Lieberman, C. Hauert, M. A. Nowak, Nature 433 (2005) 312.

[10] H. Ohtsuki, C. Hauert, E. Lieberman, M. A. Nowak, Nature 441 (2006) 502.

[11] T. Antal, S. Redner, V. Sood, Phys. Rev. Lett. 96 (2006) 188104.

[12] F. C. Santos, M. D. Santos, J. M. Pacheco, Nature 454 (2008) 213.

[13] J. Gore, H. Youk, A. V. Oudenaarden, Nature 459 (2009) 253.

[14] M. Doebeli, N. Knowlton, Proc. Natl. Acad. Sci. USA 95 (1998) 8676.

[15] T. Killingback, M. Doebeli, N. Knowlton, Proc. R. Soc. B 266 (1999) 1723.

[16] R. Albert, A. L. Barábasi, Rev. of Mod. Phys. 74 (2002) 47.

[17] F. C. Santos, J. M. Pacheco, Phys. Rev. Lett. 95 (2005) 098104.

[18] C. Hauert, J. Theor. Biol. 240 (2006) 627.

[19] A. Cassar, Games \& Econ. Behav. 58 (2007) 209.

[20] X. Thibert-Plante, L. Parrott, Complexity 12 (2007) 22.

[21] G. Abramson, M. Kuperman, Phys. Rev. E 63 (2001) 030901.

[22] L. H. Shang, X. Li, X. F. Wang, Eur. Phys. J. B 54 (2006) 369.

[23] F. Fu, L. H. Liu, L. Wang, Eur. Phys. J. B 56 (2007) 367.

[24] M. A. Nowak, R. M. May, Int. J. Bifurcat. Chaos 3 (1993) 35.

[25] J. M. Pacheco, A. Traulsen, M. A. Nowak, Phys. Rev. Lett. 97 (2006) 258103.

[26] F. Fu, T. Wu, L. Wang, Phys. Rev. E 79 (2009) 036101

[27] J. Pena, H. Volken, Phys. Rev. E 80 (2009) 016110.

[28] C. P. Roca, J. A. Cuesta, A. Sanchez, Phys. Rev. Lett. 97 (2006) 158701.

[29] Z. X. Wu, P. Holme, Phys. Rev. E 80 (2009) 026108.

[30] F. C. Santos, J. F. Rodrigues, J. M. Pacheco, Proc. R. Soc. B 273 (2006) 51.

[31] L. X. Zhong, D. F. Zheng, B. Zheng, C. Xu, P. M. Hui, Europhys. Lett. 76 (2006) 724.

[32] W. X. Wang, J. Ren, G. R. Chen, B. H. Wang, Phys. Rev. E 74 (2006) 056113.

[33] W. X. Wang, J. H. Lü, G. R. Chen, P. M. Hui, Phys. Rev. E 77 (2008) 046109.

[34] B. J. Kim, A. Trusina, P. Holme, P. Minnhagen, J. S. Chung, M. Y. Choi, Phys. Rev. E 66 (2002) 021907.

[35] L. L. Jiang, D. Y. Hua, J. F. Zhu, B. H. Wang, T. Zhou, Eur. Phys. J. B 65 (2008) 251.

[36] F. W. S. Lima, T. Hadzibeganovic, D. Stauffer, Physica A 388 (2009) 4999.

[37] C. P. Zhu, S. J. Xiong, Y. J. Tian, N. Li, K. S. Jiang, Phys. Rev. Lett. 92 (2004) 218702.

[38] S. W. Son, B. J. Kim, H. Hong, H. Jeong, Phys. Rev. Lett. 103 (2009) 228702.

[39] G. Palla, I. J. Farkas, P. Pollner, I. Derényi, T. Vicsek, New J. Phys. 9 (2007) 186

[40] F. P. Fernandes, F. W. S. Lima, J. A. Plascak, Computer Phys. Comm. 181 (2010) 1218.

[41] A. Traulsen, D. Semmann, R. D. Sommerfeld, H. J. Krambeck, M. Milinski, Proc. Natl. Acad. Sci. USA 107 (2010) 2962.

[42] S. Weber, M. Porto, Eur. Phys. J. B 69 (2009) 599.

[43] T. Qiu, C. F. Fu, G. Chen, Int. J. Mod. Phys. B 22 (2008) 4909.

[44] G. Szabó, C. Töke, Phys. Rev. E 58 (1998) 69.

[45] D. H. Zanette, Phys. Rev. E 65 (2002) 041908.

[46] D. Vilone, C. Castellano, Phys. Rev. E 69 (2004) 016109.

[47] K. Gao, W. X. Wang, B. H. Wang, Physica A 380 (2007) 528.

[48] J. Wu, R. Axelrod, J. Conflict Resolut. 39 (1995) 183.

[49] F. W. S. Lima, K. Malarz, Int. J. Modern Phys. C 17 (2006) 1273.

[50] T. Hadzibeganovic, D. Stauffer, C. Schulze, Ann. New York Acad. Sci. 1167 (2009) 221.

[51] D. Helbing, W. Yu, Proc. Natl. Acad. Sci. USA 107 (2010) 5265.

[52] Y. C. Ni, C. Xu, P. M. Hui, N. F. Johnson, Physica A 388 (2009) 4856.

[53] D. Helbing, W. Yu, Proc. Natl. Acad. Sci. USA 106 (2009) 3680.

[54] W. B. Du, X. B. Cao, M. B. Hu, H. X. Yang, H. Zhou, Physica A 388 (2009) 2215.

[55] R. Sugden, The Economics of Rights, Co-operation and Welfare, Black- well, Oxford, UK, 1986

[56] A. D. Sanchez, J. M. Lopez, M. A. Rodriguez, Phys. Rev. Lett. 88 (2002) 048701 .

[57] R. S. Sun, D. Y. Hua, Chinese Phys. Lett. 26 (2009) 086403.

[58] D. Stauffer, M. Hohnisch, S. Pittnauer, Physica A 370 (2006) 734.

[59] S. Kirkpatrick, C. D. Gelatt, M. P. Vecchi, Science 220 (1983) 671.

[60] J. M. Kumpula, J. P. Onnela, J. Saramäki, J. Kertész, K. Kaski, Computer Phys. Comm. 180 (2009) 517.

[61] J. Karpínska, K. Malarz, K. Kulakowski, Int. J Mod. Phys. C 15 (2004) 1227.

[62] K. Malarz, D. Stauffer, Adv. Complex Syst. 11 (2008) 165.

[63] K. Malarz, Theory Biosci. 125 (2007) 147. 